\title{
Meditation as a Promising Tool to Alleviate Task-oriented Frustration
}

\author{
Tianshuang Qiu ${ }^{1}$ \\ ${ }^{1}$ Eureka Research Program, United States of America \\ Correspondence: Tianshuang Qiu, Eureka Research Program, United States of America. E-mail: \\ balancein0and1@gmail.com
}

$\begin{array}{lcc}\text { Received: Sep. 28, } 2018 & \text { Accepted: October 9, } 2018 & \text { Online Published: November 26, } 2018 \\ \text { doi:10.5539/mas.v12n12p228 } & \text { URL: https://doi.org/10.5539/mas.v12n12p228 }\end{array}$

\begin{abstract}
In this research, I looked to understand how meditation could reduce task-oriented frustration. I designed two games, one easy and one difficult, to induce frustration. Using an EEG sensing headband (the Muse), I tracked the participants' brainwaves to understand the effects of meditating when frustrated. I discovered that meditating increases participants' average accuracy when playing the difficult game; I also found that meditation makes participants more methodical, taking longer to consider their choices before making a play in the game.
\end{abstract}

Keywords: meditation, frustration, task, EEG, Muse headband

\section{Introduction}

Frustration is defined as "the feeling of being upset or annoyed, especially because of the inability to change or achieve something" [1]. Therefore, it can be assumed that frustration is often felt when people fail to accomplish a task. In 1939, 6 psychologists developed the Frustration Aggression Theory, in which the psychologists theorize that "the existence of frustration always leads to some form of aggression" [2]. Because frustration can cause aggression to others, understanding the ways that we could regulate frustration is an important area of study.

Mindfulness meditation is a particularly promising approach to support individuals in managing their frustration. Meditation is a tool for people to clear their minds and for people to be fully present in the moment. Meditation has the potential to regulate mood [3]. Therefore, I hypothesize that through meditation, one could alleviate his/her frustration and perform better at stressful tasks.

\subsection{Prior Works}

In 2016, Bashivan, Rish, and Heisig, gathered data by using Muse, an EEG sensing headband, to recognize mental states [4]. Participants watched videos that are either "emotional" or "logical". The researchers trained a machine learning model using the data, and discovered that the model could be trained to recognize samples with an accuracy of $75 \%$. Similar to my research, this study also uses Muse to track the participants' brainwaves.

In 2001, Barnes, Treiber, and Davis worked with 35 participants on the topic of stress and meditation [5]. Barnes aimed to examine the effects of stress reduction methods (in this case, meditation) on the blood pressure of people who usually have high blood pressure. The participants are from local high schools who have high blood pressure, and they meditated 15 minutes twice a day. After a two-month period, Barnes concluded that compared to the control group which did not meditate, the participants had a greater decrease in blood pressure during a stress test (car driving). This proves that meditation does have a positive effect on teens with high blood pressure when under stress. My research also looks into the relationship between meditation and stress; however, I use brainwaves to track the results instead of blood pressure.

In 2014, Przybylski, Deci, Rigby and Ryan examined the hypothesis that violence in video games lead to aggression [6]. He worked with 99 university undergraduates, playing violent and non-violent games, and conducted an aggression test through a hostility scale. Przybylski found that compared to the presence of violence, whether the player was successful in his or her games is more important in deciding the aggressiveness after playing the video games. This proves that frustration with difficult video games is a good way to induce stress. I also used hard video games to induce frustration.

In this research, to simulate task-oriented frustration, I created 2 video games, one easy, one hard. Participants were asked to play both games. The easy game is a representation of a normal task, and the hard game a representation of a difficult one. The hard game induces frustration. Then half the participants are asked meditate 
to try and calm themselves. To judge the effects of meditation, I utilized an EEG sensing device: Muse headband, to monitor the participants' brainwaves. As a whole, this research is aimed to advance understandings of how meditation might ameliorate task-oriented frustration.

\section{Methods}

\subsection{Tools Used}

\subsubsection{Games}

The games, developed with Unity Engine and C\#, aim to induce frustration in participants. The goal of the games is to stop a red needle when it is on the highlighted area. For the easy version, the needle spins clockwise at a constant pace (See Figure 1). Each time the spacebar is pressed, the C\# script checks if the needle is on the highlighted area and records if the user has won or lost. The highlighted area then randomly rotates to another area of the circle, and the needle starts spinning again, thus the game continues.

A harder version of the game was developed where the highlighted area was shortened (See Figure 2), and a latency period (a slight delay between the player's action and the game's response) was added to induce frustration. After the player presses the spacebar, the hard version of the program waits for a random time between 200 to 300 milliseconds and then checks if the needle is on the highlighted area (the needle still moves during the wait). This leads to more instances of lost games, because the needle often exits the highlighted area during the wait, thereby inducing frustration. Further difficulty to the game is added by having the needle rotates in a random direction at a random speed whenever the spacebar is pressed and the highlighted area shifts to a new location.
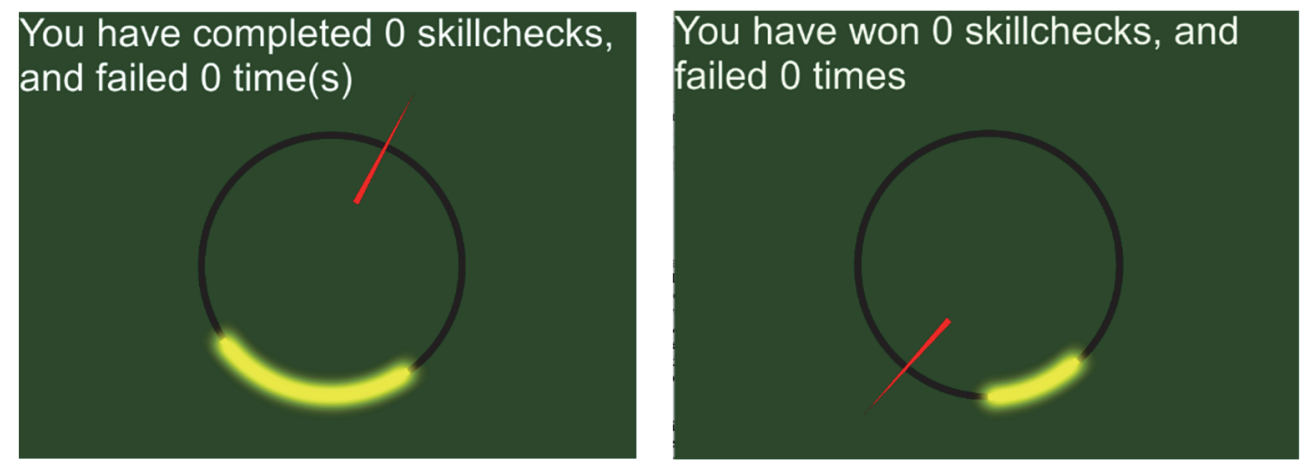

Figure 1

Figure 2

\subsubsection{Muse Headband}

The Muse headband is an EEG-sensing device with four electrodes, developed by Interaxon. It is proven to be an effective tool for analyzing brain signals. EEG stands for electroencephalogram. It is a tool that measures the electrical fluctuations by attaching electrodes to the scalp. It is widely used in the medical field, from diagnosing brain tumors to treating epilepsy [7].

However, medical EEG utilizes gel electrode pads, which are not portable. The Muse headband is a new product that is commercially available, lightweight and inexpensive. Utilizing dry electrodes, the Muse headband gives back live feedback on your mental state and is developed to help people meditate better.

\subsubsection{Muse App}

The Muse App is also developed by Interaxon. Receiving Bluetooth signals from the headband, the Muse App analyzes the different waves and generates a reading of the calmness of the wearer. When starting a meditation session, the application needs to calibrate for approximately 45 seconds to set a standard for calmness. The standard would then be used to judge whether the user is doing a good job on calming his/her mind. The Muse App responds 
in time to the mental state, the more active, the louder the app is, and vice versa.

\subsubsection{Muse Monitor}

The Muse Monitor is a third party program developed by James Clutterbuck [9]. This program can connect via Bluetooth to the Muse headband, and stream raw EEG values, including alpha, beta, gamma, delta and theta waves.

\subsection{Research Procedure}

Participants included 21 individuals, with ages ranging from 16 to 74. Most of the subjects are currently working a full-time day job. Participants are first asked to play the easy game, while I use Muse Monitor to collect data. This process would last 60 seconds. Following, the subjects would keep playing the easy game while I turn on the Muse app and start the calibration process. Afterwards, the subjects play the hard game for 180 seconds while I collect data with the Muse Monitor.

Subsequently, the subjects would be randomly chosen to perform a task of meditation, where they are asked to clear their thoughts (The subjects would play the easy game while the Muse app calibrates). When calibration completes, the group that performs meditation would be asked to meditate, while the other group proceeds to the final procedure as a control. While meditating, the Muse app would have its volume turned up so that the subjects could get live feedback on their performance regarding meditation.

For the final procedure, the subjects are asked to play the hard game again for 120 seconds.

\subsection{Data Collected}

In addition to EEG data, the following data was collected:

- Gender

- Age

- Their scores on all of the games they played (The number of times they won and lost)

- $\quad$ The graphs graphed by the Muse app

- If the subject presses the spacebar with excessive force (if so, the type of game that they did so)

- $\quad$ Surveys on their mental states

The subjects are asked to self-evaluate their frustration level, with 1 being very calm, and 5 being very frustrated. They are asked at the following times (in chronological order): when they first came in (before the experiment begins), when they complete the easy game, when they finish the hard game (after 120s), when they finish the hard game a second time (after using the Muse Monitor for 60s), when they finish meditating (applicable only to those who did meditation), when they finish the experiment.

\section{Results}

\subsection{Accuracy vs Game Type}

The average accuracy measured by $\mathrm{A}=$ Number of Successful Tries/ Total Amount of Tries when playing the easy game is $81.33 \%$; the average accuracy for the hard game is $16.63 \%$. See Figure 3 and 4 for the accuracy plotted for each participant.

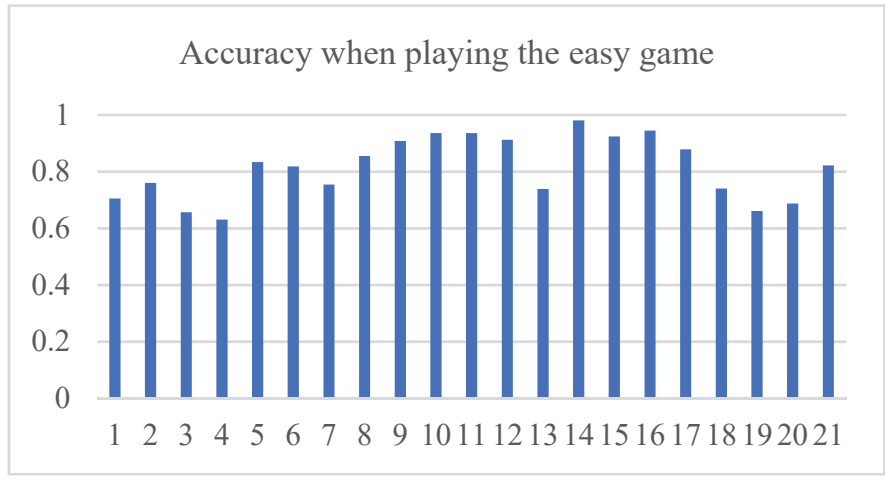

Figure 3

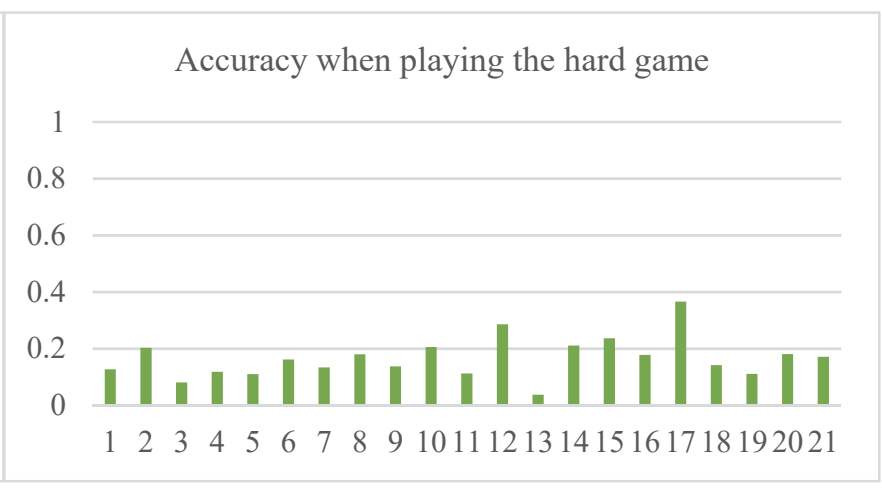

Figure 4 
When playing the hard game, the average accuracy for the difficult game increased by $7 \%$ after meditation for the meditation group from their first tries; whereas for control group, the accuracy dropped by $0.42 \%$.

In Figure 5, the orange line plots the average accuracy when participants first played, and the average accuracy when they played a second time after meditation. The blue line plots the average accuracy of the control group for their first and second games.

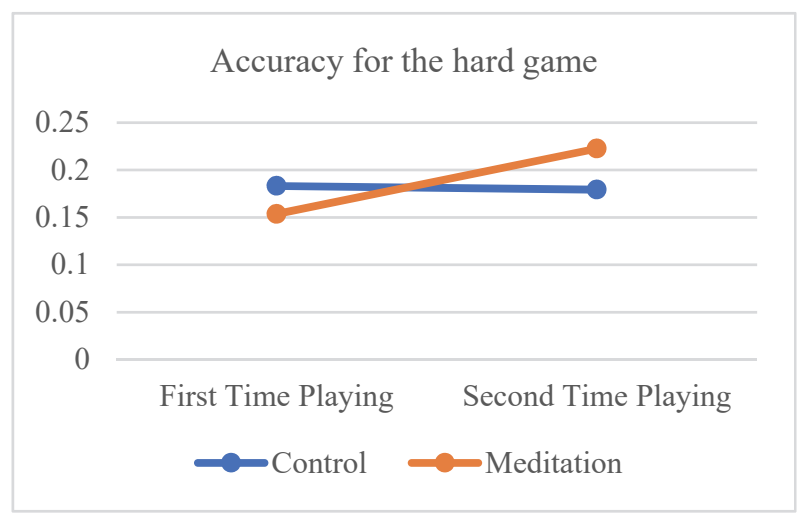

Figure 5 .

\subsection{Frequency of Key Hits}

The frequency $\mathrm{f}$ is calculated by $\mathrm{f}=$ (Total number of keystrokes)/ (The time (in seconds) of the session). The Meditation group averaged 0.75 keystrokes/second before the meditation, and 0.64 afterwards. The control group averaged 0.92 keystrokes/second their first try, and 0.85 the second. The meditation group saw a steeper decrease compared to the control group as illustrated in Figure 6.

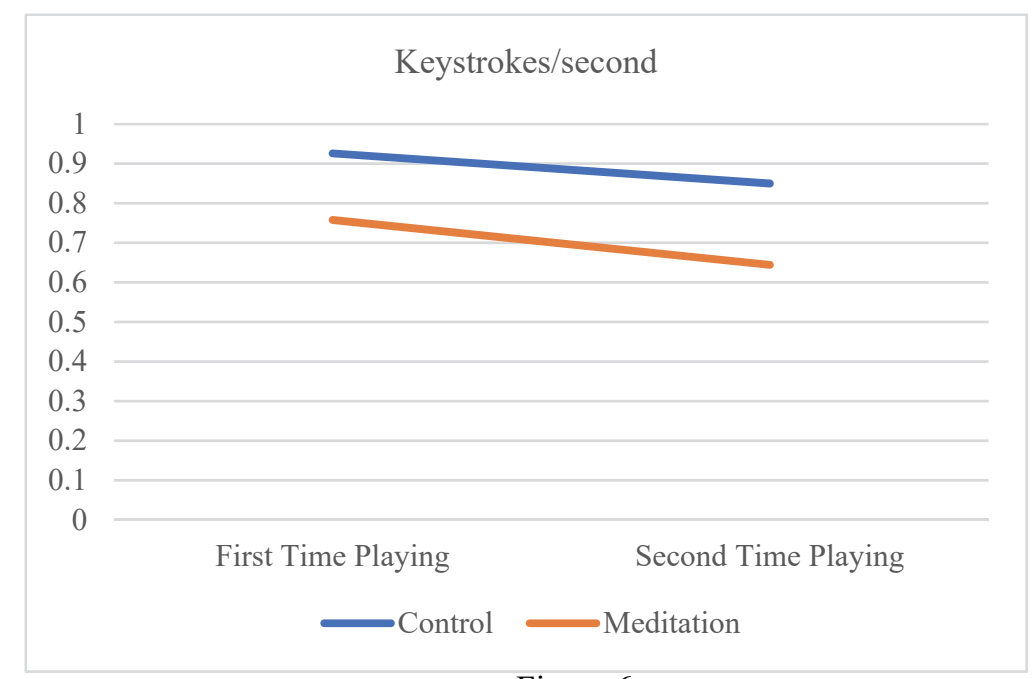

Figure 6

\subsection{Extra Findings}

When I analyzed the results, I came across some relatively interesting pieces that are significant.

\subsubsection{Cross Gender comparison}

Of the 21 participants in this study, there were 14 males, taking up $66.67 \%$ of the total participants. However, among the participants who strike the keys with excessive force, $80 \%$ were males (See Figure 7). 

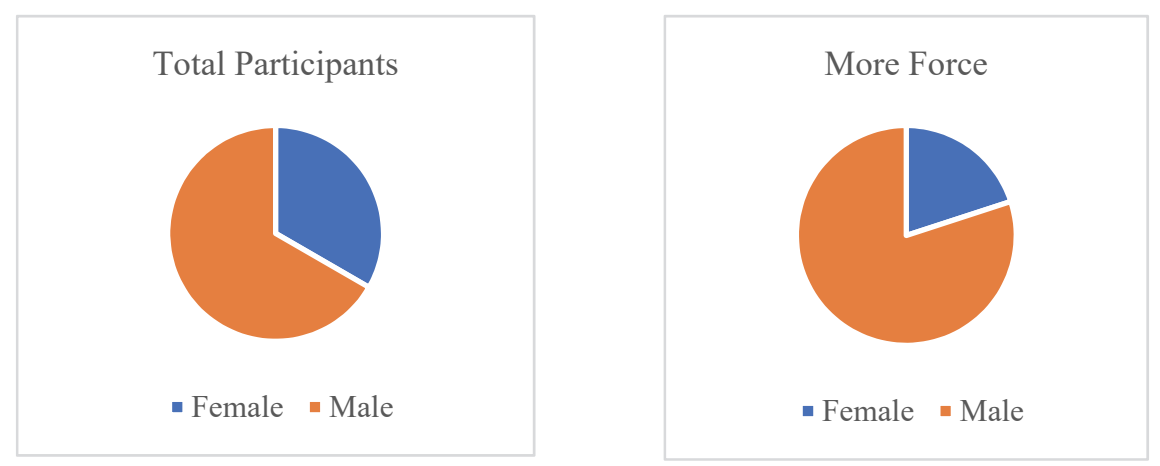

Figure 7

\subsubsection{Cross Age Accuracy Comparison for the Hard Game}

The data for when subjects play the difficult game for the first time are quite dispersed (See Figure 8). This indicates that there is no strong correlation between age and the accuracy when playing a stressful game.

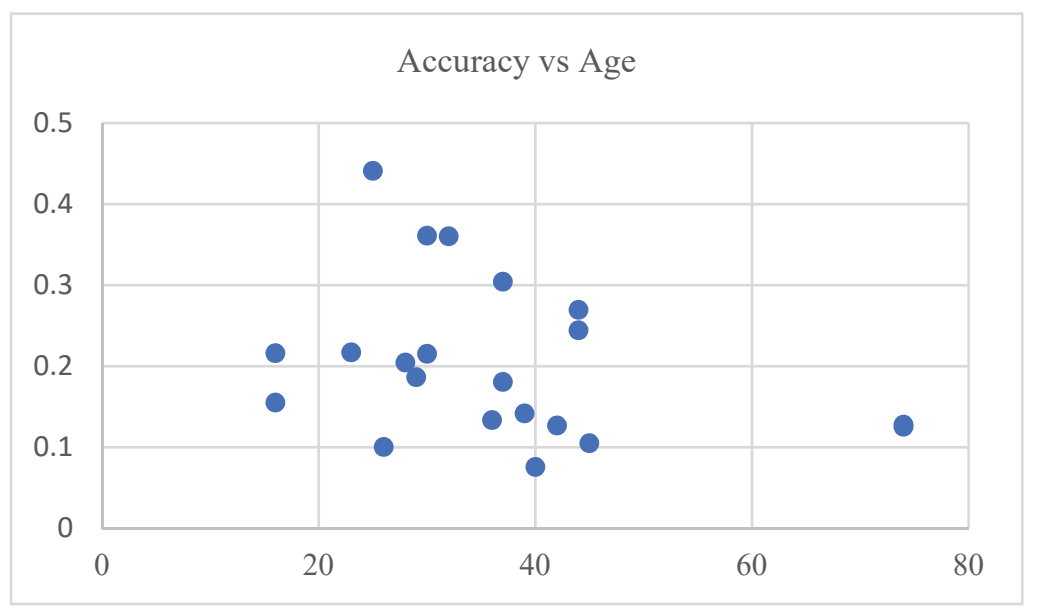

Figure 8

\section{Discussion}

The findings suggest that there is a very strong correlation between meditation and improvement in a difficult video game. The hard game was extremely frustrating to the subjects, proven by the steep decrease in accuracy and reports from the participants: roughly half of the subjects strike the key with excessive force while playing the hard game. $85.7 \%$ of the subjects expressed frustration at the seemingly "unresponsiveness" of the game, with 4 of them commenting that they were "sure that they had hit the key while it was on there (the highlighted area)."

After having ensured the success of my induction of frustration, I can further analyze the performances of subjects under this stressful situation. When playing the hard game for the second time, the accuracy for the meditation group increased by $7 \%$, far superior to the $0.42 \%$ decrease of the control group. Therefore, it is safe to conclude that meditation has a positive impact on performance under stress. This is possibly due to the calming nature of meditation, allowing the subjects to become less frustrated with their performance, and achieving a better score when they attempted again. The meditation group's average frequency decreased by 0.11 keystrokes per second after meditating, which is slightly greater than the control group's decrease of 0.07 keystrokes per second. This indicates that those who meditated seem to be more methodical, taking longer in between attempts. This could mean that for office workers, meditation is a highly useful tool to keep focused on tasks throughout the day. Because workers are more accurate and methodical after meditation, company owners could apply the findings of this research and allow workers to have mindful moments in order to increase focus. 
This research primarily focused on task-oriented frustration where subjects have a simple goal and the learning process is relatively simple. Stressful tasks generally extend beyond the simple press of a button; therefore, future researchers could attempt to look into different kinds of frustration, such as frustration related to something being hard to learn (e.g. playing the piano). This research only lasted for around 10 minutes for each participant, which again, is not representative of the long strenuous tasks that office workers often perform. It could be worthwhile for researchers to track subjects over time, and observe the effects of meditation on frustration in the long-term.

Based on the results of the research, it could be concluded that meditation does have a positive impact on performance under stress, regardless of age. Meditating also appear to make people more methodical and careful when making a decision. I propose that if we could recognize frustration, then it could be possible to perform meditation and possibly prevent aggression from frustration. Below is the work that I have done on training a machine learning algorithm to recognize "calm" and "frustrated" EEG data.

The Muse Monitor outputs raw EEG data (voltage readings from each electrode). The data could be used to predict whether the data points were from a "calm" state (playing the easy game), or the "frustrated" state(playing the hard game). I used R and the xgboost library. Since the participants were asked to report on their frustration level, with 5 representing "very frustrated", and 1 representing "very calm". To normalize the data to be between 0 and 1, I divided their self-reports by 5 . The average after playing the easy game is 0.3214 , the average after the hard game is 0.61 . Utilizing the binary classifier based on a logistic regression model, I labeled the "calm" data as 0.3214 , and the "frustrated" data as 0.61 .

Then I separated the data into 5 equal groups. The R program uses 4 of the 5 to train the model, and uses the last to evaluate how well the model has done. $\mathrm{R}$ was able to modify the model to report a $34.7 \%$ error rate. This result indicate that it is hard to adjust a model for all the participants.

If future researchers are able to achieve a higher accuracy rate, assessing your frustration levels with the Muse would become easier. For employees who work under high-stress environments, they might be susceptible to higher levels of frustration, which could lead to aggression. If employers are able to calibrate the Muse to detect frustration for each member of staff and ask them to meditate when frustrated, overall efficiency could be increased and probable aggression prevented.

\section{References}

Andrew, K., Przybylski, Edward, L., Deci, C., Scott, R., \& Richard, M. R. (2014). Competence-impeding electronic games and players' aggressive feelings, thoughts, and behaviors. https://doi.org/10.1037/a0034820

Developer Kit (2017). At Muse: The Brain Sensing Headband. Retrieved from http://developer.choosemuse.com/tools/

Dollard, J., Neal, E., Miller, L. W., Doob, Orval Hobart Mowrer, \& Robert, R. S. (1939). Frustration and aggression. https://doi.org/10.1037/10022-000

EEG Electroencephalogram - Mayfield Clinic. Retrieved from https://mayfieldclinic.com/pe-eeg.htm

frustration | Definition of frustration in English by Oxford Dictionaries. Retrieved from https://en.oxforddictionaries.com/definition/frustration

Mental State Recognition via Wearable EEG” Pouya Bashivan, Irina Rish, Steve Heisig. Jun 5, 2016. https://doi.org/arXiv:1602.00985

Mindfulness: How it could help you be happier, healthier ... - CNN.com." 15 Feb. 2017

Muse, M. (2018). Developed by James Clutterbuck. Retrieved from https://musemonitor.com/Chart.php

Vernon, A., B., Frank, A. T., \& Harry, D. (2001). Impact of Transcendental Meditation on cardiovascular function at rest and during acute stress in adolescents with high normal blood pressure. https://doi.org/10.1038/ajh.2007.65

\section{Copyrights}

Copyright for this article is retained by the author(s), with first publication rights granted to the journal.

This is an open-access article distributed under the terms and conditions of the Creative Commons Attribution license (http://creativecommons.org/licenses/by/4.0/). 